\title{
Lie Algebraic Approach to $\tau$-Functions and its Equations
}

G. POST

Dept of Applied Mathematics, Twente University of Technology, P.O. Box 217, 7500 AE Enschede, The Netherlands

(Received: 25 November 1985)

Abstract. A general construction of equations satisfied by the components of $\tau$-functions is given by considering the tensor products of modules. As an example, the homogeneous basic realization of $\hat{A}_{1}$ is given, leading to NLS equations.

\section{Introduction}

Some years ago, Date, Jimbo, Kashiwara, and Miwa [2,6] showed that solutions of many soliton equations can be considered as group orbits of highest weight vectors $\mathbf{V}_{\Lambda}$ of $L(\Lambda)$.

Kac [7] gave a Lie algebraic interpretation for the case that the representation $L(\Lambda)$ remains irreducible when considered as an 1 -module. Here $s$ is a Heisenberg subalgebra. One can extend this construction to the case where $L(\Lambda)$ does not remain irreducible as an 1 -module.

Modified equations can be found by considering $L(\Lambda) \otimes L(\tilde{\Lambda})(\Lambda \neq \tilde{\Lambda})$ instead of $L(\Lambda) \otimes L(\Lambda)$.

\section{Irreducible Highest Weight Modules}

We start with a given affine Kac-Moody algebra $g(A)$ with a $(n+1) \times(n+1)$ matrix $A$, where $g(A)$ has the generators $e_{0}, \ldots, e_{n}$ and $f_{0}, \ldots, f_{n}$ and a center $c$. The principal grading $\delta$ is defined by

$$
\delta\left(e_{i}\right)=-\delta\left(f_{J}\right)=1 \quad(i, j=0 \ldots n)
$$

We suppose that we have a Heisenberg subalgebra 1 in $g(A)$ with a basis $\left\{p_{i}, q_{j}, c\right\}$ $(i, j>0)$ such that

$$
\left[p_{i}, q_{j}\right]=\delta_{i j} c \quad(i, j \in \mathbb{N}, i, j>0) .
$$

We also suppose that $\delta\left(p_{i}\right)=-\delta\left(q_{i}\right)>0$.

Take an integrable irreducible highest weight module $L(\Lambda)$ (over $g(A))$ with highest weight vector $V_{\Lambda}$, and denote by $\Pi$ the representation of $g(A)$ it affords. One has $\Pi(c)=m \cdot \operatorname{Id}($ cf. Lemma 9.3 of [7]). 
We define the grading $\delta_{L}$ on $L(\Lambda)$ by

$$
\delta_{L}\left(\Pi(g) \mathbf{V}_{\Lambda}\right)=-\delta(g) \quad(g \in \mathscr{U}(g(A)) \cdot) .
$$

Because $\delta\left(p_{i}\right)>0, \Pi\left(p_{i}\right)$ is locally nilpotent on $L(\Lambda)$. The highest weight module $L(\Lambda)$ splits up as a direct sum of the irreducible 1 -modules, all isomorphic to each other (see Lemma 14.4 of [7]):

and

$$
L(\Lambda) \simeq \underset{i \in I}{\oplus} R \otimes_{\mathbb{C}} u_{i}, \quad R=\mathbb{C}\left[x_{1}, x_{2}, \ldots\right]
$$

$$
\Pi\left(p_{i}\right)=m \frac{\partial}{\partial x_{i}} \otimes 1 ; \quad \Pi\left(q_{i}\right)=x_{i} \otimes 1 ; \quad \Pi(c)=m \cdot \text { Id } .
$$

$1 \otimes u_{i}$ are the vacuum vectors of the irreducible components with eigenvalue $m$.

The $g(A)$-module $L(\Lambda)$ carries a unique nondegenerate contravariant Hermitian form $H_{1}$ such that $H_{1}\left(\mathbf{V}_{\Lambda}, \mathbf{V}_{\Lambda}\right)=1$ (see Lemma 11.5 of [7]).

\section{The $\tau$-Functions and Equations}

To make $\tau$-functions, one has to identify a group $G$ associated to $g(A)$. For example, one could take the group generated by $\exp \left(t e_{i}\right)$ and $\exp \left(t f_{i}\right)(i=0 \ldots n, t \in \mathbb{C})$. The $\tau$-function $\tau(g)$ is defined by

$$
\tau(g)=\Pi(g) \mathbf{V}_{\Lambda} \quad(g \in G) .
$$

To find equations satisfied by $\tau(g)$, one considers $L(\Lambda) \otimes L(\Lambda)$. This module is completely reducible ( $\$ 10.7$ of [7]). The module generated by $\mathbf{V}_{\Lambda} \otimes \mathbf{V}_{\Lambda}$ is denoted by $L_{\text {high }}$ and the submodule $L_{\text {high }}^{\perp}$ by $L_{\text {low }}$. In general, $L_{\text {low }}$ is not irreducible.

Orthogonality is taken with respect to $H$ defined by

$$
H\left(\mathrm{~V}_{1} \otimes W_{1}, \mathrm{~V}_{2} \otimes W_{2}\right)=H_{1}\left(\mathrm{~V}_{1}, \mathrm{~V}_{2}\right) \cdot H_{1}\left(W_{1}, W_{2}\right)
$$

It is now essential that $\tau(g) \otimes \tau(g) \in L_{\text {high }}$, from which, by definition, follows

$$
H(\mathrm{~V} \otimes W, \tau(g) \otimes \tau(g))=0 \quad\left(\mathrm{~V} \otimes W \in L_{\text {low }}\right) .
$$

This equation will lead to bilinear equations under one more assumption. We assume that

$$
-\omega_{0}\left(q_{i}\right)=a_{i} p_{i} \quad\left(a_{i} \in \mathbb{C}, \omega_{0} \text { anti-linear Cartan involution }\right) .
$$

According to (1.1), we can write $v=\Sigma P_{i}(x) u_{i}\left(v \in L(\Lambda)\right.$, we omit $\left.\otimes_{C}\right)$ and also $\tau(g)=\Sigma \tau_{k}(x) u_{k}$. For $w \in L(\Lambda) \otimes L(\Lambda)$ we write

$$
w=\sum_{i, j} P_{i}\left(x^{(0)}\right) u_{i} \otimes Q_{j}\left(x^{(1)}\right) u_{j}=\sum_{i, j} P_{i}\left(x^{(0)}\right) Q_{j}\left(x^{(1)}\right) u_{i} \otimes u_{j}
$$

Suppose

$$
H_{1}\left(u_{i}, u_{j}\right)=\varepsilon_{i j} \text { and } \tilde{D}_{x}=\left(a_{1} \frac{\partial}{\partial x_{1}}, a_{2} \frac{\partial}{\partial x_{2}}, \ldots\right) .
$$


Using (2.3), one finds

$$
H_{1}\left(\sum_{i} P_{i}(x) u_{i}, \sum_{j} Q,(x) u_{j}\right)=\left.\sum_{i, J} \varepsilon_{i j} \bar{P}_{i}\left(m \tilde{D_{x}}\right) Q_{j}(x)\right|_{x=0}
$$

Changing variables

$$
2 x_{j}=x_{j}^{(0)}+x_{j}^{(1)} \text { and } 2 y_{j}=x_{j}^{(0)}-x_{j}^{(1)}
$$

leads to

$$
\Pi \otimes \Pi\left(p_{i}\right)=m \frac{\partial}{\partial x_{i}} ; \quad \Pi \otimes \Pi\left(q_{j}\right)=2 x_{j} ; \quad \Pi \otimes \Pi(c)=2 m \cdot \mathbf{I d}
$$

Using these variables, $L_{\text {low }}$ can be written as $L_{\text {low }}=\mathbb{C}[x] \otimes$ Hir, where the elements of Hir are of the form

$$
\sum_{i, j} P_{i j}(y) u_{i} \otimes u_{j} \in \operatorname{Hir} \Leftrightarrow Q(x) \sum_{i, j} P_{i j}(y) u_{i} \otimes u_{j} \in L_{\text {low }} .
$$

Substituting this in (2.2), and using (2.4) and the arbitrariness of $Q$, yields

$$
\left.\sum_{i, j, k, l} \bar{P}_{i j}\left(m \tilde{D_{y}}\right) \tau_{k}(x+y) \tau_{l}(x-y) \varepsilon_{i k} \varepsilon_{j l}\right|_{y=0}=0 .
$$

These are equations in Hirota's bilinear form. The sums on $i$ and $j$ are finite and, therefore, so are the sums on $k$ and $l$, since $\varepsilon_{i k} \neq 0$ for only finitely many $k$ ( $i$ fixed). We have assumed the analycity of $\tau$.

\section{Example}

As an example, we give the homogeneous basic realization of $\hat{A}_{1}$ (see [5]). So we take $g(A)=\hat{A}_{1}$ and $p_{i}=\frac{1}{2} h \otimes t^{i}, q_{j}=(1 / j) h \otimes t^{-j}$, and $\Lambda=\Lambda_{0}$. Then $a_{j}=2 / j$ (see (2.3)) and $m=1$ (see (1.1)).

One can find $u_{i}$ so that

$$
\begin{aligned}
& L\left(\Lambda_{0}\right) \simeq \sum_{i \in \mathbb{Z}} R \otimes_{\mathbb{C}} u_{i} ; \quad \delta_{L}\left(u_{i}\right)=2 i^{2}-i ; \\
& 1 \otimes u_{0}=\mathbf{V}_{\Lambda_{0}} ; \quad H_{1}\left(u_{i}, u_{j}\right)=\delta_{i j} .
\end{aligned}
$$

Moreover,

$$
\begin{aligned}
& \Pi\left(e \otimes t^{\prime}\right) Q(x) u_{k}=(-1)^{k+1} \sum_{m} p_{m-i-2 k-1}(x) p_{m}(-\tilde{D}) Q(x) u_{k+1}, \\
& \Pi\left(f \otimes t^{i}\right) Q(x) u_{k}=(-1)^{k} \sum_{m} p_{m-i+2 k-1}(-x) p_{m}(\tilde{D}) Q(x) u_{k-1},
\end{aligned}
$$

where $p_{l}(x)$ is defined by

$$
\sum p_{l}(x) k^{l}=\exp \left[\sum_{i \geqslant 1} x_{i} k^{i}\right]
$$


For $\tau_{0}, \tau_{1}$, and $\tau_{-1}$, one finds (among others) the following three equations

$$
\begin{aligned}
& D_{1}^{2}\left(\tau_{0} \cdot \tau_{0}\right)+2 \tau_{1} \cdot \tau_{-1}=0 ; \quad\left(D_{1}^{2}+D_{2}\right)\left(\tau_{0} \cdot \tau_{1}\right)=0 ; \\
& \left(D_{1}^{2}+D_{2}\right)\left(\tau_{-1} \cdot \tau_{0}\right)=0 .
\end{aligned}
$$

Introducing $\tau_{1} / \tau_{0}=q$ and $\tau_{-1} / \tau_{0}=q^{*}$ yields

$$
q_{t}=q_{x x}-2 q^{2} q^{*} ; \quad-q_{t}^{*}=q_{x x}^{*}-2 q^{*^{2}} q .
$$

\section{Remarks}

(1) By considering two different modules $L(\Lambda)$ and $L(\tilde{\Lambda})$, both realized in the 'same' way, one can derive modified equations. For example, the principal realizations of $L\left(\Lambda_{0}\right)$ and $L\left(\Lambda_{1}\right)$ lead to the MKdV (after considering $L\left(\Lambda_{0}\right) \otimes L\left(\Lambda_{1}\right)$ ) (cf. [9]). In the same way, the homogeneous realizations of $L\left(\Lambda_{0}\right)$ and $L\left(\Lambda_{1}\right)$ lead to the NLS with a derivative coupling (cf. [10]), that is, Equation (3.1) with $q q_{x}\left(q^{*} q_{x}^{*}\right)$ instead of $q^{2}\left(q^{* 2}\right)$.

(2) It is already known that some connections exist between the NLS equation and the Lie algebra $\hat{A}_{1}$ (cf. $[1-4,6]$ ).

The approach followed here tries to explain, using only Lie algebras, how the equations for the $\tau$-functions arise. This derivation is quite different from $[2,6]$. In those papers, the equations are consequences of a bilinear identity for the wavefunctions $w$ and $w^{*}$, while in this Letter the equations reflect the orthogonality relations for $\tau$-functions.

This also opens possibilities for attacking other algebras (for example $\hat{E}_{8}$ ). Our approach is an extension of $\mathrm{Kac}$ [7, Ch. 14] where irreducible s-modules are treated.

Wakimoto and Yamada [11] suggest yet another interesting possibility by extending the algebra with a Virasoro algebra.

Drinfeld and Sokolov [3] associate a hierarchy of KdV-like equations to every vertex of a Dynkin diagram. This Dynkin diagram belongs to a Kac-Moody Lie algebra. An interesting problem is to investigate the relation between their approach (leading to Hamiltonian forms for the equations) and the $\tau$-function approach [8].

Finally, the Estabrook-Wahlquist prolongation method applied to the NLS equation leads to a prolongation algebra closely connected with $\hat{A}_{1}[4]$.

\section{Acknowledgement}

I would like to express my appreciation to Professor R. Martini for a number of helpful discussions.

\section{References}

1. Ablowitz, M. J., Kaup, D. J., Newell, A. C., and Segur, H., Stud. Appl. Math. 53, 249 (1974).

2. Date, E., Jimbo, M., Kashiwara, M., and Miwa, T., 'Transformation Groups for Soliton Equations', 
Proceedings of RIMS Symposium on Non-Linear Integrable Systems-Classical Theory and Quantum Theory, World Science Publ., Singapore, 1983.

3. Drinfeld, V. G. and Sokolov, V. V., Sov. Math. Dokl. 23, 457 (1981).

4. Van Eck, H. N., Gragert, P. K. H., and Martini, R., Proc. Ned. Akad. Wetensch. Ser. A 86, 165 (1983).

5. Frenkel, I. B. and Kac, V. G., Inv. Math. 62, 23 (1980).

6. Jimbo, M. and Miwa, T., 'Solitons and Infinite Dimensional Lie Algebras', RIMS-439, Kyoto University, 1983.

7. Kac, V. G., Infinite Dimensional Lie Algebras, Progress in Math. Vol. 44, Birkhäuser, 1983.

8. Newell, A. C., Solitons in Mathematics and Physics, SIAM, Philadelphia, 1985.

9. Post, G., 'Lie Algebraic Approach to the mod-KdV', to appear J. Math. Phys. March 1986.

10. Post, G., 'Lie Algebraic Approach to the NLS', Memorandum 531, Appl. Math., Twente University, 1985.

11. Wakimoto, M. and Yamada, H., Lett. Math. Phys. 7, 513 (1983). 